\title{
Salvianolic Acid B, a Potential Chemopreventive Agent, for Head and Neck Squamous Cell Cancer
}

\author{
Yuan Zhao, ${ }^{1}$ Yinhan Guo, ${ }^{2}$ and Xinbin $\mathbf{G u}^{1}$ \\ ${ }^{1}$ College of Dentistry, Howard University, 600 W Street, NW, Washington, DC 20059, USA \\ ${ }^{2}$ Oriental TenGen Technology Development Co. Ltd., Department of D\&R, P.O. Box 100078, Beijing 100078, China
}

Correspondence should be addressed to Xinbin Gu, xgu@howard.edu

Received 1 September 2010; Accepted 21 November 2010

Academic Editor: Rengaswamy Sankaranarayanan

Copyright ( 2011 Yuan Zhao et al. This is an open access article distributed under the Creative Commons Attribution License, which permits unrestricted use, distribution, and reproduction in any medium, provided the original work is properly cited.

\begin{abstract}
Head and neck squamous cell cancer (HNSCC) is one of the top ten cancers in the United States. The survival rate of HNSCC has only marginally improved over the last two decades. In addition, African-American men bear a disproportionate burden of this preventable disease. Therefore, a critical challenge of preventive health approaches is warranted. Salvianolic acid B (Sal-B) isolated from Salvia miltiorrhiza Bge, which is a well-know Chinese medicines has been safely used to treat and prevent aging diseases for thousand of years. Recently, the anticancer properties of Sal-B have received more attention. Sal-B significantly inhibits or delays the growth of HNSCC in both cultured HNSCC cells and HNSCC xenograft animal models. The following anticancer mechanisms have been proposed: the inhibition of COX-2/PGE-2 pathway, the promotion of apoptosis, and the modulation of angiogenesis. In conclusion, Sal-B is a potential HNSCC chemopreventive agent working through antioxidation and antiinflammation mechanisms.
\end{abstract}

\section{HNSCC and Chemoprevention}

Over $90 \%$ of head and neck cancers are squamous cell carcinomas (HNSCC). Oral cancer accounts for a major proportion of HNSCC, which is the sixth most common cancer worldwide. In the United States, oral and pharyngeal cancers alone are diagnosed in about 36,540 Americans annually, and 7,880 are projected to die from these diseases in 2010 according to the American Cancer Society. HNSCC has been less studied compared to other cancers and the incidence of this cancer has not shown any improvement in the last 20 years (Figure 1). The 5-year survival rate for oral and pharyngeal cancers in Caucasian patients is $56 \%$, while for African American men; it is only 34\% [1]. In over $50 \%$ of first diagnosed cases of HNSCC in African American men, the cancer has already metastasized to other organs, such as the lungs. HNSCC prevention, earlier detection, and viable treatment options are of paramount importance to reduce the cancer incidence, improve patient outcomes and diminish the disparity.

HNSCC have been considered to be a typical multistep carcinogenesis with stepwise accumulations of genetic alterations resulting in aberrant cellular appearance, deregulated cell growth and carcinoma [2]. Patients with early stages of disease still have high risk to develop a second malignancy. A normal epithelial cell can take many years to undergo the multiple cellular and genetic alterations that lead to malignant changes. Thus, it remains an appealing strategy to develop effective, nontoxic and affordable novel pharmacological agents for preventing development of HNSCC and second primary HNSCC [2-5]. Chemoprevention has been considered a rational and appealing strategy to prevent or delay the development of HNSCC, additionally; dietary nutrients such as green tea, $\beta$-carotene and vitamin $\mathrm{E}$ have been also used as preventive agents [5-8]. Extensive studies have suggested that green tea, one of the most commonly consumed beverages worldwide, can reduce the risk of HNSCC development by inducing antioxidative activity via apoptosis and inhibiting epidermal growth factor receptor related signaling pathway $[7,9,10]$. There have been an increased number of case reports that high doses of green tea beverages cause hepatotoxicity [11]. Both vitamin $\mathrm{E}$ and $\beta$-carotene revealed the growth-inhibitory effect against lung cancer in cell culture and rodent models. But the promising activities have not translated into clinical success. Indeed, these supplements may actually 
lead to unexpected detrimental effects in humans as well as beneficial effects $[12,13]$. Hence, the crux is to find an effective, nontoxic and affordable novel pharmacological agent in clinical trials for preventing carcinogenesis and the development of HNSCC as well as second primary HNSCC.

\section{Salvianolic Acid B}

Radix Salviae miltiorrhizae (danshen or tanshen), the dried root of Salvia miltiorrhiza Bge is very important and popular in traditional Chinese medicine. It that has been widely and successfully used treating and preventing aging diseases, such as cardiovascular and cerebrovascular diseases, and cancers for thousand years and is ranked as a "Super grade" drug recorded in Shen-Nung's Pen-Ts'ao [14]. Currently, danshen has been accepted and used in Japan, the United States and some European countries [14-16]. In the last 50 years, Danshen received more attention by modern scientists that more than 70 compounds, including the hydrophilic depsides derivatives and the lipophilic diterpenoids, have been isolated from the Danshen herb [16, 17]. Salvianolic acid B (Sal-B) is the most abundant and bioactive member of the hydrophilic components in Danshen. Therefore, Sal-B is used as a quality control ingredient and active marker for S. miltiorrhiza Bge products by the National Pharmacopoeia Council of China. Sal-B contains seven phenolic hydroxyls which have been found to be closely related to redox potentials and/or antioxidant activities [18]. The structure of Sal-B is depicted in Figure 2. Sal-B has been studied for its preventive effects against cancer as well as cardiovascular, neurodegenerative, and other diseases [19-23]. The mechanisms mainly contribute to its antiinflammatory and antioxidative properties, modulation of apoptosis, inhibition of platelet aggregation, improved coronary microcirculation, as well as, regulation of angiogenic processes [14, 24, 25]. We will introduce the function and biological activities of Sal-B, validate its efficacy on HNSCC, and discuss the foreground of this component.

\section{Antiinflammatory Activities}

It appears that there is a general concept that chronic inflammation characterized by continued active inflammation responses and tissue destruction, can be a major cause of cancers and occur during the aging process [26-28]. Mounting studies have reported that Sal-B is capable of preventing the development of cancer; and the possible antiinflammatory mechanisms of Sal-B involve modulating cytokines, Cyclooxygenase-2/prostaglandin E2 (COX$2 / \mathrm{PGE}-2)$ pathway $[24,25,29], \mathrm{NF}-\kappa \mathrm{B}[30-32]$, TNF- $\alpha$ [3335] and MMPs [36-38]. Numbers of clinical experiences indicate its effectiveness and safety in contrast to the disadvantages of nonsteroidal antiinflammatory drugs (NSAIDs). NSAIDs, used primarily to treat inflammation, are associated with several serious side effects including gastrointestinal discomfort, cardiovascular disease and kidney failure [3942].
3.1. COX-2/PGE-2. COX-2, an inducible form of cyclooxygenase is undetectable in most normal tissues, but abundant in the pathogenesis of inflammatory and neoplastic diseases. Additionally, its principal metabolite PGE2 has pleiotropic effects such as promoting cell proliferation, inhibiting cell death, promoting tumor angiogenesis, and decreasing immunosurveillance. Sal-B has been reported to attenuate significantly COX-2 expression and PGE2 production with or without lipopolysaccharide (LPS)-induced both in vitro and in vivo $[24,25,29]$, and which may be attributed to the downregulation of JNK and ERK phosphorylation and blockage of MAPKs phosphorylation [29].

3.2. NF- $\kappa B$. NF- $\kappa \mathrm{B}$, a protein complex that controls the transcription of DNA, regulates cellular responses as a "first responder" to harmful cellular stimuli, and its aberrant expression is linked to cancer and inflammation [43]. Several experiments clarified that the antiinflammatory effects of Sal-B depend on the inhibition of the NF- $\kappa \mathrm{B}$ signaling pathway [30-32]. Moreover, Sal-B attenuates the expression of VCAM-1 and ICAM-1 in TNF- $\alpha$ stimulated human aortic endothelial cell by partial blockage of NF- $\kappa$ B expression [34].

3.3. TNF- $\alpha$. Tumor necrosis factor-alpha (TNF- $\alpha$ ), a representative proinflammatory cytokine damages cell structure and increases endothelial permeability and is involved in systemic inflammation [44]. Sal-B has showed to significantly reduced the production of TNF- $\alpha$ induced by LPS treatment in rat primary microglia in a dose-dependent manner [32]. In addition, Sal-B protects endothelial cell against TNF- $\alpha$ disruption by inhibiting vascular endothelial growth factor (VEGF) and extracellular signal-regulated kinase (ERK) activation [45].

3.4. MMPs. Matrix metalloproteinases (MMPs), inflammatory mediators are expressed in vascular cells in the course of atherosclerosis [46]. Sal-B treatment effectively inhibits MMP-2 and MMP-9 activitation and expression both cell culture and animal models, and it also, downregulates JNK and ERK phosphorylation [36, 37]. Some researchers found Sal-B could suppress high glucose-induced mesangial cells proliferation and extracellular matrix production in a dose-dependent manner, partially through modulating the cell-cycle progress and MMP-2 and MMP-9 activities $[30,31]$.

\section{Antioxidative Activities}

A vast amount of evidence suggests that overproduction of reactive oxygen species (ROS) and reactive nitrogen species (RNS) can damage cellular lipids, inhibit the normal function of proteins or DNA, and are associated with the pathogenesis of atherosclerosis, cardiovascular diseases, hypertension, ischemia/reperfusion injury, neurodegenerative diseases, and cancer [47, 48]. Sal-B, as an antioxidant neutralizes direct ROS attacks and terminates free radicalmediated oxidative reactions to protect the human body from such diseases [49]. 
4.1. Radical Scavenging. Reactive oxygen species (ROS), such as superoxide anion, hydroxyl radicals and hydrogen peroxide are chemically reactive molecules derived from oxygen. They usually contain one or more unpaired electrons in the atomic or molecular orbital [50]; therefore, they can easily participate in redox reactions and they play a crucial role in biological systems [51, 52]. Excess ROS can disturb the equilibrium status of prooxidant/antioxidant reactions, leading to the disruption of cellular functions in contrast to low/moderate concentrations that occur in response to induction of a mitogenic reaction and normal function of several cellular signaling pathways $[53,54]$. There is increasing evidence that Sal-B has the capability to scavenging free radicals including superoxide anion, hydroxyl, DPPH (1,1-diphenyl-2-picryl-hydrazyl) and ABTS (2-azino-bis(3ethylbenzthiazoline-6- sulfonic acid)) radicals in addition to hydrogen peroxide due to redox properties of the phenolic structure [55]. Sal-B showed a high antioxidant capacity in terms of neutralizing free radicals, as well as exhibiting significantly higher scavenging activity than 1-Ascorbic acid (vitamin C) [56, 57]. 50\% radical scavenging activity at a concentration of the Sal-B lower $\sim 50 \%$ and $\sim 40 \%$ than Vitamin C in DPPH and ABTS assays, respectively (1.81 \pm 0.01 versus $3.44 \pm 0.03 \mu \mathrm{g} / \mathrm{ml}, 1.43 \pm 0.01$ versus $2.50 \pm$ $0.02 \mu \mathrm{g} / \mathrm{ml}$ ) [57].

4.2. Antioxidant Activities. Free radicals have been elucidated to cause oxidative damage to cellular components, including attacks on DNA, oxidation of proteins and production of lipid peroxidation, these processes lead to disorder in cellular, tissue and organ function $[52,58,59]$. Sal-B has been reported to be a powerful and effective antioxidant, not only reducing lipid peroxidation, but also rescuing the loss of antioxidant enzyme activities against fibrosis and ischemia-reperfusion injuries $[60,61]$. Some studies have reported that Sal-B can protect the brain and heart from ischemia-reperfusion injury by improving the recovery of motor function via regulating energy metabolism and maintaining the balance of free radicals such as SOD, GSH, and ATP levels against lipid peroxidation and superoxide anion production [62-65]. In the hepatic stellate cells (HSCs), Sal-B exerts suppressive effects on ROS to inhibit the proliferation and lipid peroxidation of HSCs through inhibiting NADPH oxidase and TGF- $\beta 1$ secretion $[49,66$, 67]. Studies have shown that ROS leads to the oxidation of low-density lipoproteins and accumulates within plaques and contributes to the atherosclerosis $[66,68]$. Sal-B was identified to be a potent antioxidant, endothelial-protecting agent, an inhibitor that suppresses the expression of ICAM and VCAM, capable of inhibiting LDL oxidation and also inhibits ox-LDL induced endothelial injuries [69]. In sum, the antioxidative properties of Sal-B are closely associated with its protective effect of aging diseases [70].

\section{HNSCC and Sal-B}

Cancers have a close and delicate relationship with inflammation $[26,71,72]$. Inflammation often exists in the tumor microenvironment and is induced by inflammatory mediators produced by the tumor [73-75]. HNSCCs are highly inflammatory and aggressive, and they overexpress a number of inflammatory mediators such as COX-2, EGFR, VEGF and MMPs [76-78]. Sal-B, as chemopreventive agent exerts its effects by inhibiting tumor initiation and development; its anticarcinogenic activities have been clearly demonstrated in both cell cultures and animal models [24]. Furthermore, research has also shown that the combining Sal-B with other preventive agents is more effective than single-agent chemoprevention [25].

We tested the anticancer function of Sal-B in five human HNSCC cell lines (JHU-6, JHU-11, JHU-13, JHU-22 and JHU-29) that Sal-B significantly inhibit the cell growth in cultured cells [24]. In the animal experiments, HNSCC solid tumor volume in Sal-B treated group were significantly lower than those in untreated control groups [24]. The outcome is consistently obtained in human breast and prostate cancer cell lines. We found that Sal-B selectively suppresses COX-2-related mRNA and protein expression instead of housekeeping enzyme of COX-1 in the presence or absence of LPS stimulation. It seems that the chemopreventive effects of Sal-B depend on COX-2 expression levels, the higher the expression of COX-2 the more sensitive is Sal-B HNSCC cell growth-inhibition and PGE2 reduction. In addition, Sal$\mathrm{B}$ induced caspase-dependent apoptosis by cleavage of a caspase substrate, poly (ADP) ribose polymerase (PARP). Sal-B also decreased the cellular amounts of antiapoptotic proteins such as NF- $\kappa \mathrm{B}, \mathrm{MDM}-2, \mathrm{Bcl}-2$ and $\mathrm{Bcl}-\mathrm{xL}$ and increased proapototic proteins such as p53 and caspase 3 $[24,79]$. The mechanism of cancer-prevention was attributed to the COX-2/PGE2 inhibition and apoptotic pathway induction. PGE2, one of important prostaglandin product of COX-2 is involved in chronic inflammation [80].

A promising strategy to enhance the cancer-preventive efficacy is to use two anticancer agents in combination, which may produce synergy and lower the dose required for each agent $[79,81]$. Celecoxib, a selective COX-2 inhibitor has been reported to have cancer-preventive effects in various types of cancers including HNSCC [82]. However, it was found to be associated with a dose-dependent cardiovascular morbidity that limited its long-term use. Hence, we decided to use Sal-B combined with low-dose celecoxib in order to increase the anticancer efficacy and reduce drug side effects. The outcome showed that the combination of halfdose of Sal-B and celecoxib greatly enhanced the inhibition of HNSCC cell proliferation compared with either Sal$\mathrm{B}$ or celecoxib alone both in cell culture (JHU-06, JHU011, JHU-013 and JHU-022) and tumor xenografts. The combination was associated with profound inhibition of the COX-2/PGE2/EGFR pathways, enhanced induction of apoptosis, and reduced the side effects of celecoxib due to dose reduction at the same time [25].

The anticancer effects of Sal-B were also found in 7,12dimethylbenz-[a] anthracene-(DMBA) induced oral carcinogenesis in hamsters [83]. Experiments showed that Sal-B treatment significantly decreased the oral cancer incidence. Antiangiogenesis may be one of the possible mechanisms of inhibiting malignant transformation of oral precancerous 


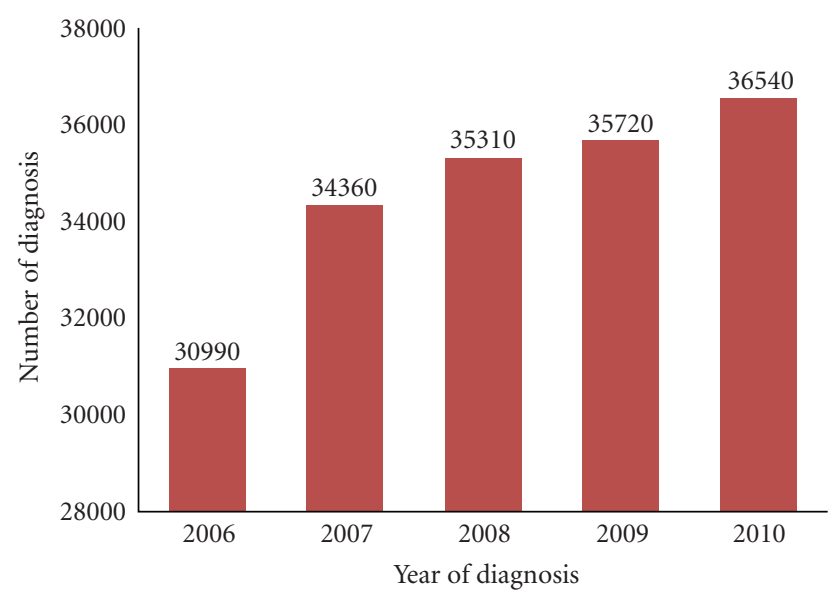

FIGURE 1: Incidence of oral cavity and pharynx cancer.

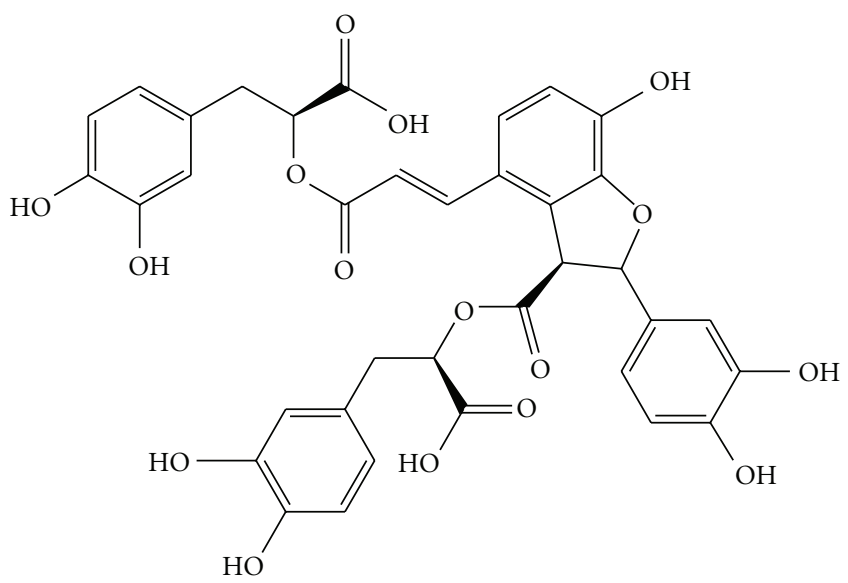

Figure 2: Chemical structure of Salvianolic acid B.

$[75,84]$. The formation of microvessels, and the expression of proangiogenic factors HIF $1 \alpha$ and VEGF, was inhibited by Sal-B.

Recently, we were inclined to accept the concept that the prevention is better than a cure. Actually, prevention is more valuable to reduce the incidence of HNSCC than increase survival rate $[85,86]$. Anticancer properties of Sal$\mathrm{B}$ are able to prevent and delay the malignant conversion of premalignant lesions and/or cell growth via inhibition of inflammation and angiogenesis and reduction of apoptosis (Figure 3).

\section{Problem and Future Prospects}

Sal-B as a popular compound of Traditional Chinese Medicine has been studied for its preventive effects against HNSCC. Most of the proposed beneficial effects have been attributed to antioxidative and antiinflammatory effects. It is known that the relationships among oxidation, inflammation and cancer are considered to be extremely complex. Firstly, chronic inflammation increase the risk of developing many types of cancer including HNSCC, and inflammatory

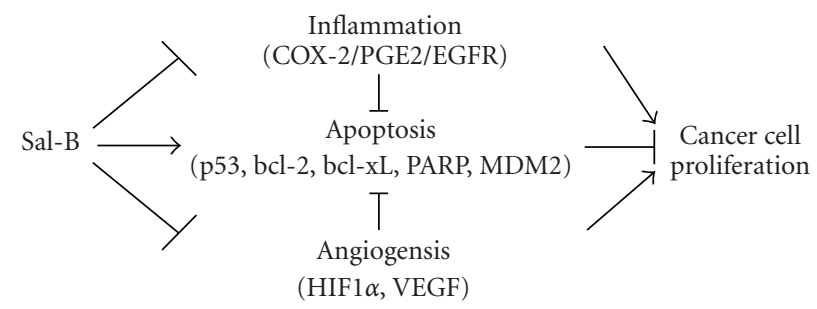

Figure 3: Possible anticancer activites of Salvianolic acid B.

cells, chemokines and cytokines are present in the microenvironment of all tumors [71]. Secondly, ROS, an endogenous class of carcinogens trigger the mutation of the cells have been demonstrated in the principal step of carcinogenesis and contribute to cancer progression. Moreover, cancer cells frequently produce more ROS than normal cells $[87,88]$. Thirdly, activated inflammatory cells generate ROS and reactive RNS in response to proinflammaory stimuli, which can function as chemical effectors in inflammation-driven carcinogenesis; ROS-induced oxidations are implicated in inflammation via regulation signal pathways and related enzymes such as COX-2 [88, 89]. Sal-B, a natural anticarcinogenic agent with antiantioxidant and antiinflammatory activities, reacts easily with free radicals and inhibits effectively COX-2/PGE-2 pathway as well as regulating related cell signal pathways. It is difficult to distinguish which is determines prevention to HNSCC because oxidation, inflammation and cancer are intertwined in a complex web. Sal-B has been showed a significant advantage for the prevention and treatment in HNSCC due to effectiveness and nontoxic nature.

The discovery of Sal-B anticancer properties is followed a path of from "bed" to "bench". A thousand years clinical practices demonstrate that Danshen is able to effectively and safely prevent and treat aging diseases, such as cardiovascular diseases and cancers. The mechanism studies of Danshen have been concerned until latest 50 years. Sal-B is a most abundant and bioactive member of hydrophilic components in Danshen. The same as Danshen, Sal-B is a safer agent with no major side effects [20]. Due to our aging society, both cancer and cardiovascular diseases have increasingly become the two major killers and human health hazards. In recent years, the amount of cytotoxic agents and targeted therapies used to treat HNSCC, include classic chemotherapeutic agents, chemoprevention agents such as COX-2 inhibitors, monoclonal antibodies targeting tyrosine kinase receptors, small molecule tyrosine kinase inhibitors, and antiangiogenic drugs [90]. Unfortunately, these result in cardiovascular toxicity. Especially, celecoxib, a selective COX-2 inhibitor has been required restricted use owing to its potential to effects the cardiovascular system in longterm use by the Food and Drug Administration and was announced the early cessation of a cancer-prevention clinical trial. It is hailed that Sal-B is a not only cancer-preventive agent but an also cardiovascular protective agent. Sal-B inhibits cancer cell proliferation in vitro and in vivo in addition to regulating the microenvironment. In a study with 
celecoxib, Sal-B resulted in platelet aggregation, playing a key role in cardiovascular protection [20]. Since Sal-B exerts dual pharmacy characteristics, it will lead to broad applications in the future. But related-mechanisms are still not fully understood, we need specific and profound studies about Sal-B.

In addition, some studies also reported Sal-B was released from nanotechnology samples faster and had increased antioxidant activity compared to the traditionally-powdered samples [89, 91, 92]. Combined with chemopreventive agents of traditional Chinese Medicine will further promote development of Sal-B.

We found an interesting and confusing phenomenon that Sal-B showed a distinct attack and protective features in different cell lines. Some studies reported Sal-B protected the SH-SY5Y neuroblastoma cells, hepatocyte and bone marrow stem cells against apoptosis by relieving oxidative stress and modulating the apoptotic process [93-95]; on the other hand, Sal-B has been revealed to activate apoptosis pathways in order to inhibit cancer cell proliferation $[24,25]$. It seems to be controversial; whereas, the mechanism of signal regulation is very complex and therefore the role of Sal$B$ depends on the type of cell line, the microenvironment. Different concentrations of ROS showed both the induction and inhibition in cancer development. To define an accurate mechanism of Sal-B, particularly which are Sal-B exact targets, still calls for more studies and developments.

In conclusion, Sal-B, a natural antiinflammatory (selective COX-2 inhibitor) and antioxidative agent has chemopreventive activity on HNSCC; due to its effectiveness and safety it could have much more commercial value for food and medicine purposes.

\section{Acknowledgments}

This work was supported in part by Grant P20 CA118770 from the National Cancer Institute and Tianjue Gu Foundation.

\section{References}

[1] D. E. Morse and A. R. Kerr, "Disparities in oral and pharyngeal cancer incidence, mortality and survival among black and white Americans," Journal of the American Dental Association, vol. 137, no. 2, pp. 203-212, 2006.

[2] S. M. Lippman, J. Sudbø, and W. K. Hong, "Oral cancer prevention and the evolution of molecular-targeted drug development," Journal of Clinical Oncology, vol. 23, no. 2, pp. 346-356, 2005.

[3] D. T. Lin, K. Subbaramaiah, J. P. Shah, A. J. Dannenberg, and J. O. Boyle, "Cyclooxygenase-2: a novel molecular target for the prevention and treatment of head and neck cancer," Head and Neck, vol. 24, no. 8, pp. 792-799, 2002.

[4] G. J. Kelloff, S. M. Lippman, A. J. Dannenberg et al., "Progress in chemoprevention drug development: the promise of molecular biomarkers for prevention of intraepithelial neoplasia and cancer-a plan to move forward," Clinical Cancer Research, vol. 12, no. 12, pp. 3661-3697, 2006.

[5] X. Gu, X. Song, Y. Dong et al., "Vitamin E succinate induces ceramide-mediated apoptosis in head and neck squamous cell carcinoma in vitro and in vivo," Clinical Cancer Research, vol. 14, no. 6, pp. 1840-1848, 2008.

[6] J. D. Lambert and R. J. Elias, "The antioxidant and prooxidant activities of green tea polyphenols: a role in cancer prevention," Archives of Biochemistry and Biophysics, vol. 501, no. 1, pp. 65-72, 2010.

[7] Y. C. Lim, S. H. Lee, M. H. Song et al., "Growth inhibition and apoptosis by (-)-epicatechin gallate are mediated by cyclin D1 suppression in head and neck squamous carcinoma cells," European Journal of Cancer, vol. 42, no. 18, pp. 3260-3266, 2006.

[8] A. K. Sakhi, S. K. Bøhn, S. Smeland et al., "Postradiotherapy plasma lutein, $\alpha$-carotene, and $\beta$-carotene are positively associated with survival in patients with head and neck squamous cell carcinoma," Nutrition and Cancer, vol. 62, no. 3, pp. 322$328,2010$.

[9] M. Masuda, M. Suzui, J. T. E. Lim, A. Deguchi, J. W. Soh, and I. B. Weinstein, "Epigallocatechin-3-gallate decreases VEGF production in head and neck and breast carcinoma cells by inhibiting EGFR-related pathways of signal transduction," Journal of Experimental Therapeutics and Oncology, vol. 2, no. 6, pp. 350-359, 2002.

[10] M. Masuda, M. Suzui, and I. B. Weinstein, "Effects of epigallocatechin-3-gallate on growth, epidermal growth factor receptor signaling pathways, gene expression, and chemosensitivity in human head and neck squamous cell carcinoma cell lines," Clinical Cancer Research, vol. 7, no. 12, pp. 4220-4229, 2001.

[11] G. Mazzanti, F. Menniti-Ippolito, P. A. Moro et al., "Hepatotoxicity from green tea: a review of the literature and two unpublished cases," European Journal of Clinical Pharmacology, vol. 65, no. 4, pp. 331-341, 2009.

[12] E. N. Scott, A. J. Gescher, W. P. Steward, and K. Brown, "Development of dietary phytochemical chemopreventive agents: biomarkers and choice of dose for early clinical trials," Cancer Prevention Research, vol. 2, no. 6, pp. 525-530, 2009.

[13] O. P. Heinonen and D. Albanes, "The effect of vitamin E and beta carotene on the incidence of lung cancer and other cancers in male smokers," New England Journal of Medicine, vol. 330, no. 15, pp. 1029-1035, 1994.

[14] X. Wang, S. L. Morris-Natschke, and K. H. Lee, "New developments in the chemistry and biology of the bioactive constituents of Tanshen," Medicinal Research Reviews, vol. 27, no. 1, pp. 133-148, 2007.

[15] T. H. Tsai, "Analytical approaches for traditional Chinese medicines exhibiting antineoplastic activity," Journal of Chromatography B: Biomedical Sciences and Applications, vol. 764, no. 1-2, pp. 27-48, 2001.

[16] L. Zhou, Z. Zuo, and M. S. S. Chow, "Danshen: an overview of its chemistry, pharmacology, pharmacokinetics, and clinical use," Journal of Clinical Pharmacology, vol. 45, no. 12, pp. 1345-1359, 2005.

[17] Y. G. Li, L. Song, M. Liu, Z. B. Hu, and Z. T. Wang, "Advancement in analysis of Salviae miltiorrhizae Radix et Rhizoma (Danshen)," Journal of Chromatography A, vol. 1216, no. 11, pp. 1941-1953, 2009.

[18] E. J. Lien, S. Ren, H. H. Bui, and R. Wang, "Quantitative structure-activity relationship analysis of phenolic antioxidants," Free Radical Biology and Medicine, vol. 26, no. 3-4, pp. 285-294, 1999.

[19] J. Liu, H. M. Shen, and C. N. Ong, "Salvia miltiorrhiza inhibits cell growth and induces apoptosis in human hepatoma HepG cells," Cancer Letters, vol. 153, no. 1-2, pp. 85-93, 2000. 
[20] T. O. Cheng, "Cardiovascular effects of Danshen," International Journal of Cardiology, vol. 121, no. 1, pp. 9-22, 2007.

[21] M. K. Tang, D. C. Ren, J. T. Zhang, and G. H. Du, "Effect of salvianolic acids from Radix Salviae miltiorrhizae on regional cerebral blood flow and platelet aggregation in rats," Phytomedicine, vol. 9, no. 5, pp. 405-409, 2002.

[22] S. S. K. Durairajan, Q. Yuan, L. Xie et al., "Salvianolic acid $B$ inhibits $A \beta$ fibril formation and disaggregates preformed fibrils and protects against $\mathrm{A} \beta$-induced cytotoxicty," Neurochemistry International, vol. 52, no. 4-5, pp. 741-750, 2008.

[23] I. S. Abd-Elazem, H. S. Chen, R. B. Bates, and R. C. C. Huang, "Isolation of two highly potent and non-toxic inhibitors of human immunodeficiency virus type 1 (HIV-1) integrase from Salvia miltiorrhiza," Antiviral Research, vol. 55, no. 1, pp. 91-106, 2002.

[24] Y. Hao, T. Xie, A. Korotcov et al., "Salvianolic acid B inhibits growth of head and neck squamous cell carcinoma in vitro and in vivo via cyclooxygenase-2 and apoptotic pathways," International Journal of Cancer, vol. 124, no. 9, pp. 2200-2209, 2009.

[25] Y. Zhao, Y. Hao, H. Ji et al., "Combination effects of salvianolic acid B with low-dose celecoxib on inhibition of head and neck squamous cell carcinoma growth in vitro and in vivo," Cancer Prevention Research, vol. 3, no. 6, pp. 787-796, 2010.

[26] L. M. Coussens and Z. Werb, "Inflammation and cancer," Nature, vol. 420, no. 6917, pp. 860-867, 2002.

[27] R. Gebhardt, "Oxidative stress, plant-derived antioxidants and liver fibrosis," Planta Medica, vol. 68, no. 4, pp. 289-296, 2002.

[28] J. K. Kundu and Y. J. Surh, "Inflammation: gearing the journey to cancer," Mutation Research, vol. 659, no. 1-2, pp. 15-30, 2008.

[29] Y. L. Chen, C. S. Hu, F. Y. Lin et al., "Salvianolic acid $\mathrm{B}$ attenuates cyclooxygenase-2 expression in vitro in LPStreated human aortic smooth muscle cells and in vivo in the apolipoprotein-E-deficient mouse aorta," Journal of Cellular Biochemistry, vol. 98, no. 3, pp. 618-631, 2006.

[30] P. Luo, Z. Tan, Z. Zhang, H. Li, and Z. Mo, "Inhibitory effects of salvianolic acid B on the high glucose-induced mesangial proliferation via NF- $\kappa \mathrm{B}$-dependent pathway," Biological and Pharmaceutical Bulletin, vol. 31, no. 7, pp. 1381-1386, 2008.

[31] Y. H. Chen, S. J. Lin, Y. L. Chen, P. L. Liu, and J. W. Chen, "Anti-inflammatory effects of different drugs/agents with antioxidant property on endothelial expression of adhesion molecules," Cardiovascular and Hematological DisordersDrug Targets, vol. 6, no. 4, pp. 279-304, 2006.

[32] S. X. Wang, L. M. Hu, X. M. Gao, H. Guo, and G. W. Fan, "Anti-inflammatory activity of salvianolic acid B in microglia contributes to its neuroprotective effect," Neurochemical Research, vol. 35, no. 7, pp. 1029-1037, 2010.

[33] L. X. Xie, S. S. K. Durairajan, J. H. Lu et al., "The effect of salvianolic acid B combined with laminar shear stress on TNF$\alpha$-stimulated adhesion molecule expression in human aortic endothelial cells," Clinical Hemorheology and Microcirculation, vol. 44, no. 4, pp. 245-258, 2010.

[34] Y. H. Chen, S. J. Lin, H. H. Ku et al., "Salvianolic acid $\mathrm{B}$ attenuates VCAM-1 and ICAM-1 expression in TNF- $\alpha$ treated human aortic endothelial cells," Journal of Cellular Biochemistry, vol. 82, no. 3, pp. 512-521, 2001.

[35] Z. Zhou, Y. Liu, A. D. Miao, and S. Q. Wang, "Salvianolic acid $\mathrm{B}$ attenuates plasminogen activator inhibitor type 1 production in TNF- $\alpha$ treated human umbilical vein endothelial cells," Journal of Cellular Biochemistry, vol. 96, no. 1, pp. 109-116, 2005.
[36] Y. H. Liang, P. Li, Q. F. Huang, J. Zhao, X. Liu, and M. K. Dai, "Salvianolic acid B in vitro inhibited matrix metalloproteinases-1, -2, and -9 activities," Zhong Xi Yi Jie He Xue Bao, vol. 7, no. 2, pp. 145-150, 2009.

[37] S. J. Lin, I. T. Lee, Y. H. Chen et al., "Salvianolic acid $\mathrm{B}$ attenuates MMP-2 and MMP-9 expression in vivo in apolipoprotein-E-deficient mouse aorta and in vitro in LPStreated human aortic smooth muscle cells," Journal of Cellular Biochemistry, vol. 100, no. 2, pp. 372-384, 2007.

[38] L. Wang, Y. Tao, S. Li, G. Chen, and C. Liu, "Effects of salvianolic acid $\mathrm{B}$ on lipid peroxidation and metalloproteinase-2 activity in fibrotic liver in rat," Zhongguo Zhongyao Zazhi, vol. 35 , no. 1, pp. 71-75, 2010.

[39] P. Randelli, F. Randelli, P. Cabitza, and L. Vaienti, “The effects of COX-2 anti-inflammatory drugs on soft tissue healing: a review of the literature," Journal of Biological Regulators and Homeostatic Agents, vol. 24, no. 2, pp. 107-114, 2010.

[40] W. Jaksch, C. Dejaco, and M. Schirmer, "4 years after withdrawal of rofecoxib: where do we stand today?" Rheumatology International, vol. 28, no. 12, pp. 1187-1195, 2008.

[41] J. B. Raskin, "Gastrointestinal effects of nonsteroidal antiinflammatory therapy," American Journal of Medicine, vol. 106, no. 5, pp. 3S-12S, 1999.

[42] R. John and A. M. Herzenberg, "Renal toxicity of therapeutic drugs," Journal of Clinical Pathology, vol. 62, no. 6, pp. 505$515,2009$.

[43] T. Lawrence, "The nuclear factor NF-kappaB pathway in inflammation," Cold Spring Harbor Perspectives in Biology, vol. 1, no. 6, Article ID a001651, 2009.

[44] B. B. Aggarwal, S. Shishodia, K. Ashikawa, and A. C. Bharti, "The role of TNF and its family members in inflammation and cancer: lessons from gene deletion," Current Drug TargetsInflammation \& Allergy, vol. 1, pp. 327-341, 2002.

[45] M. Ding, T. X. Ye, G. R. Zhao, Y. J. Yuan, and Z. X. Guo, "Aqueous extract of Salvia miltiorrhiza attenuates increased endothelial permeability induced by tumor necrosis factor- $\alpha$," International Immunopharmacology, vol. 5, no. 11, pp. 16411651, 2005.

[46] K. Kessenbrock, V. Plaks, and Z. Werb, "Matrix metalloproteinases: regulators of the tumor microenvironment," Cell, vol. 141, no. 1, pp. 52-67, 2010.

[47] V. B. Djordjević, L. Zvezdanović, and V. Cosić, "Oxidative stress in human diseases," Srpski Arhiv za Celokupno Lekarstvo, vol. 136, supplement 2, pp. 158-165, 2008.

[48] C. S. Yang, J. D. Lambert, and S. Sang, "Antioxidative and anti-carcinogenic activities of tea polyphenols," Archives of Toxicology, vol. 83, no. 1, pp. 11-21, 2009.

[49] M. K. Tsai, Y. L. Lin, and YI. T. Huang, "Effects of salvianolic acids on oxidative stress and hepatic fibrosis in rats," Toxicology and Applied Pharmacology, vol. 242, no. 2, pp. 155-164, 2010.

[50] P. Evans and B. Halliwell, "Free radicals and hearing: cause, consequence, and criteria," Annals of the New York Academy of Sciences, vol. 884, pp. 19-40, 1999.

[51] C. Balsano and A. Alisi, "Antioxidant effects of natural bioactive compounds," Current Pharmaceutical Design, vol. 15, no. 26, pp. 3063-3073, 2009.

[52] M. Valko, D. Leibfritz, J. Moncol, M. T. D. Cronin, M. Mazur, and J. Telser, "Free radicals and antioxidants in normal physiological functions and human disease," International Journal of Biochemistry and Cell Biology, vol. 39, no. 1, pp. 4484, 2007.

[53] J. L. Bosmans, P. Holvoet, S. E. H. Dauwe et al., "Oxidative modification of low-density lipoproteins and the outcome of 
renal allografts at 1 1/2 years," Kidney International, vol. 59, no. 6, pp. 2346-2356, 2001.

[54] M. Valko, H. Morris, M. Mazúr, P. Rapta, and R. F. Bilton, "Oxygen free radical generating mechanisms in the colon: do the semiquinones of vitamin K play a role in the aetiology of colon cancer?" Biochimica et Biophysica Acta, vol. 1527, no. 3, pp. 161-166, 2001.

[55] Y. Cai, Q. Luo, M. Sun, and H. Corke, "Antioxidant activity and phenolic compounds of 112 traditional Chinese medicinal plants associated with anticancer," Life Sciences, vol. 74, no. 17, pp. 2157-2184, 2004.

[56] G. R. Zhao, H. M. Zhang, T. X. Ye et al., "Characterization of the radical scavenging and antioxidant activities of danshensu and salvianolic acid B," Food and Chemical Toxicology, vol. 46, no. 1, pp. 73-81, 2008.

[57] Y. Sun, H. Zhu, J. Wang, Z. Liu, and J. Bi, "Isolation and purification of salvianolic acid A and salvianolic acid B from Salvia miltiorrhiza by high-speed counter-current chromatography and comparison of their antioxidant activity," Journal of Chromatography B: Analytical Technologies in the Biomedical and Life Sciences, vol. 877, no. 8-9, pp. 733-737, 2009.

[58] K. Rahman, "Studies on free radicals, antioxidants, and cofactors," Clinical Interventions in Aging, vol. 2, no. 2, pp. 219236, 2007.

[59] M. Valko, C. J. Rhodes, J. Moncol, M. Izakovic, and M. Mazur, "Free radicals, metals and antioxidants in oxidative stressinduced cancer," Chemico-Biological Interactions, vol. 160, no. 1, pp. 1-40, 2006.

[60] G. H. Du, Y. Qiu, and J. T. Zhang, "Salvianolic acid B protects the memory functions against transient cerebral ischemia in mice," Journal of Asian Natural Products Research, vol. 2, no. 2, pp. 145-152, 2000.

[61] Y. L. Lin, C. H. Wu, M. H. Luo et al., "In vitro protective effects of salvianolic acid B on primary hepatocytes and hepatic stellate cells," Journal of Ethnopharmacology, vol. 105, no. 1-2, pp. 215-222, 2006.

[62] Y. H. Chen, G. H. Du, and J. T. Zhang, "Salvianolic acid B protects brain against injuries caused by ischemia- reperfusion in rats," Acta Pharmacologica Sinica, vol. 21, no. 5, pp. 463466,2000

[63] J. Y. Han, J. Y. Fan, Y. Horie et al., "Ameliorating effects of compounds derived from Salvia miltiorrhiza root extract on microcirculatory disturbance and target organ injury by ischemia and reperfusion," Pharmacology and Therapeutics, vol. 117, no. 2, pp. 280-295, 2008.

[64] M. Tang, W. Feng, Y. Zhang, J. Zhong, and J. Zhang, "Salvianolic acid B improves motor function after cerebral ischemia in rats," Behavioural Pharmacology, vol. 17, no. 5-6, pp. 493-498, 2006.

[65] J. Zhong, M. K. Tang, Y. Zhang, Q. P. Xu, and J. T. Zhang, "Effect of salvianolic acid B on neural cells damage and neurogenesis after brain ischemia-reperfusion in rats," Yaoxue Xuebao, vol. 42, no. 7, pp. 716-721, 2007.

[66] K. Chen and J. F. Keaney, "Reactive oxygen species-mediated signal transduction in the endothelium," Endothelium, vol. 11, no. 2, pp. 109-121, 2004.

[67] G. Yao, L. Xu, X. Wu, L. Xu, J. Yang, and H. Chen, "Preventive effects of salvianolic acid $b$ on transforming growth factor$\beta 1$-induced epithelial-to-mesenchymal transition of human kidney cells," Biological and Pharmaceutical Bulletin, vol. 32, no. 5, pp. 882-886, 2009.

[68] K. Chen, S. R. Thomas, and J. F. Keaney Jr., "Beyond LDL oxidation: ROS in vascular signal transduction," Free Radical Biology and Medicine, vol. 35, no. 2, pp. 117-132, 2003.
[69] M. S. Shiao, J. J. Chiu, B. W. Chang et al., "In search of antioxidants and anti-atherosclerotic agents from herbal medicines," BioFactors, vol. 34, no. 2, pp. 147-157, 2008.

[70] M. A. Esmaeili and A. Sonboli, "Antioxidant, free radical scavenging activities of Salvia brachyantha and its protective effect against oxidative cardiac cell injury," Food and Chemical Toxicology, vol. 48, no. 3, pp. 846-853, 2010.

[71] A. Mantovani, P. Allavena, A. Sica, and F. Balkwill, "Cancerrelated inflammation," Nature, vol. 454, no. 7203, pp. 436-444, 2008.

[72] A. Sgambato and A. Cittadini, "Inflammation and cancer: a multifaceted link," European Review for Medical and Pharmacological Sciences, vol. 14, no. 4, pp. 263-268, 2010.

[73] S. P. Hussain and C. C. Harris, "Inflammation and cancer: an ancient link with novel potentials," International Journal of Cancer, vol. 121, no. 11, pp. 2373-2380, 2007.

[74] F. Wang, P. Arun, J. Friedman, Z. Chen, and C. van Waes, "Current and potential inflammation targeted therapies in head and neck cancer," Current Opinion in Pharmacology, vol. 9, no. 4, pp. 389-395, 2009.

[75] M. M. Moore, W. Chua, K. A. Charles, and S. J. Clarke, "Inflammation and cancer: causes and consequences," Clinical Pharmacology and Therapeutics, vol. 87, no. 4, pp. 504-508, 2010.

[76] M. S. Choe, X. Zhang, H. J. C. Shin, D. M. Shin, and C. Zhuo, "Interaction between epidermal growth factor receptorand cyclooxygenase 2-mediated pathways and its implications for the chemoprevention of head and neck cancer," Molecular Cancer Therapeutics, vol. 4, no. 9, pp. 1448-1455, 2005.

[77] J. W. Kim, A. R. M. R. Amin, and D. M. Shin, "Chemoprevention of head and neck cancer with green tea polyphenols," Cancer Prevention Research, vol. 3, no. 8, pp. 900-909, 2010.

[78] G. R. Thomas, H. Nadiminti, and J. Regalado, "Molecular predictors of clinical outcome in patients with head and neck squamous cell carcinoma," International Journal of Experimental Pathology, vol. 86, no. 6, pp. 347-363, 2005.

[79] L. Lo Russo and L. Lo Muzio, "Combination chemotherapy for head and neck cancer: the addition of Bcl-2 inhibitors," Current Opinion in Investigational Drugs, vol. 10, no. 12, pp. 1325-1333, 2009.

[80] A. Greenhough, H. J. M. Smartt, A. E. Moore et al., "The COX-2/PGE pathway: key roles in the hallmarks of cancer and adaptation to the tumour microenvironment," Carcinogenesis, vol. 30, no. 3, pp. 377-386, 2009.

[81] H. Xiao and C. S. Yang, "Combination regimen with statins and NSAIDs: a promising strategy for cancer chemoprevention," International Journal of Cancer, vol. 123, no. 5, pp. 983990, 2008.

[82] D. Y. Zhang, J. Wu, F. Ye et al., "Inhibition of cancer cell proliferation and prostaglandin E synthesis by Scutellaria baicalensis," Cancer Research, vol. 63, no. 14, pp. 4037-4043, 2003.

[83] Z. T. Zhou, Y. Yang, and J. P. Ge, "The preventive effect of salvianolic acid B on malignant transformation of DMBAinduced oral premalignant lesion in hamsters," Carcinogenesis, vol. 27, no. 4, pp. 826-832, 2006.

[84] B. J. Monk, L. J. Willmott, and D. A. Sumner, "Antiangiogenesis agents in metastatic or recurrent cervical cancer," Gynecologic Oncology, vol. 116, no. 2, pp. 181-186, 2010.

[85] F. L. Queiroga, I. Pires, M. Parente, H. Gregório, and C. S. Lopes, "COX-2 over-expression correlates with VEGF and tumour angiogenesis in canine mammary cancer," Veterinary Journal. In press. 
[86] D. P. Toomey, J. F. Murphy, and K. C. Conlon, "COX-2, VEGF and tumour angiogenesis," Surgeon, vol. 7, no. 3, pp. 174-180, 2009.

[87] P. T. Schumacker, "Reactive oxygen species in cancer cells: live by the sword, die by the sword," Cancer Cell, vol. 10, no. 3, pp. 175-176, 2006.

[88] T. P. Szatrowski and C. F. Nathan, "Production of large amounts of hydrogen peroxide by human tumor cells," Cancer Research, vol. 51, no. 3, pp. 794-798, 1991.

[89] A. A. Geronikaki and A. M. Gavalas, "Antioxidants and inflammatory disease: synthetic and natural antioxidants with anti-inflammatory activity," Combinatorial Chemistry and High Throughput Screening, vol. 9, no. 6, pp. 425-442, 2006.

[90] A. Albini, G. Pennesi, F. Donatelli, R. Cammarota, S. de Flora, and D. M. Noonan, "Cardiotoxicity of anticancer drugs: the need for cardio-oncology and cardio-oncological prevention," Journal of the National Cancer Institute, vol. 102, no. 1, pp. 14$25,2010$.

[91] J. R. Liu, G. F. Chen, H. N. Shih, and P. C. Kuo, "Enhanced antioxidant bioactivity of Salvia miltiorrhiza (Danshen) products prepared using nanotechnology," Phytomedicine, vol. 15, no. 1-2, pp. 23-30, 2008.

[92] Q. Peng, T. Gong, J. Zuo, J. Liu, D. Zhao, and Z. Zhang, "Enhanced oral bioavailability of salvianolic acid B by phospholipid complex loaded nanoparticles," Pharmazie, vol. 63, no. 9, pp. 661-666, 2008.

[93] B. Lu, Z. Ye, Y. Deng, H. Wu, and J. Feng, "MEK/ERK pathway mediates cytoprotection of salvianolic acid B against oxidative stress-induced apoptosis in rat bone marrow stem cells," Cell Biology International, vol. 34, no. 11, pp. 1063-1068, 2010.

[94] X. Yan, T. Zhou, Y. Tao, Q. Wang, P. Liu, and C. Liu, "Salvianolic acid $\mathrm{B}$ attenuates hepatocyte apoptosis by regulating mediators in death receptor and mitochondrial pathways," Experimental Biology and Medicine, vol. 235, no. 5, pp. 623$632,2010$.

[95] G. Zeng, T. Tang, H.-J. Wu et al., "Salvianolic acid b protects SH-SY5Y neuroblastoma cells from1-methyl-4phenylpyridinium-induced apoptosis," Biological and Pharmaceutical Bulletin, vol. 33, no. 8, pp. 1337-1342, 2010. 


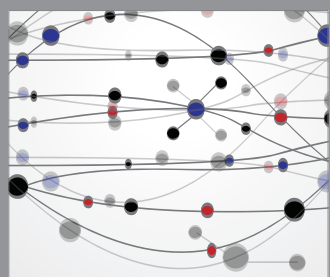

The Scientific World Journal
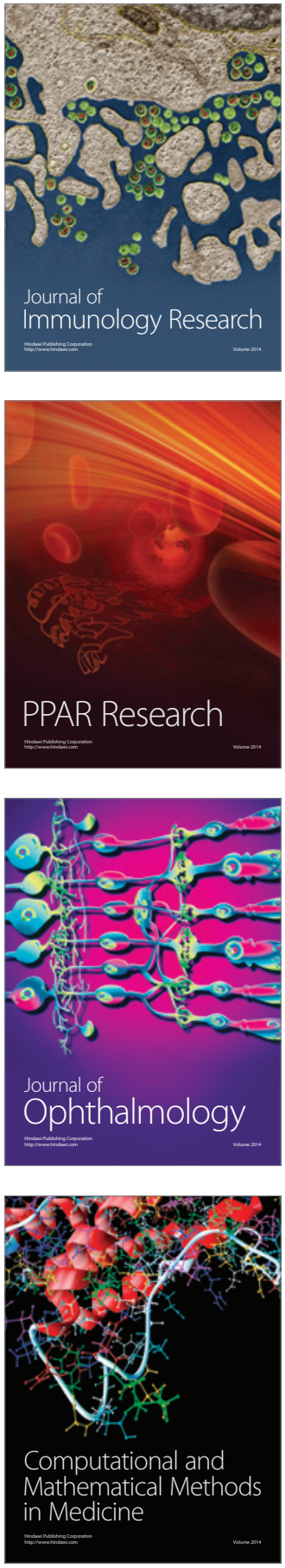

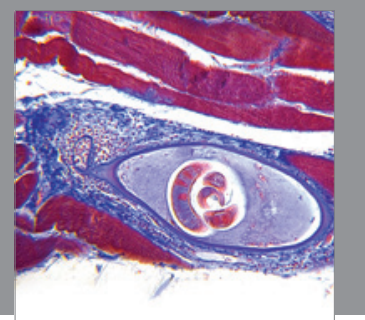

Gastroenterology

Research and Practice
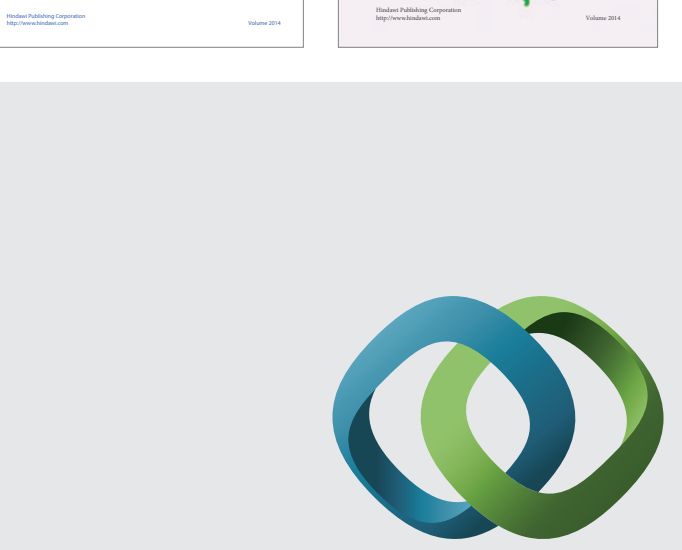

\section{Hindawi}

Submit your manuscripts at

http://www.hindawi.com
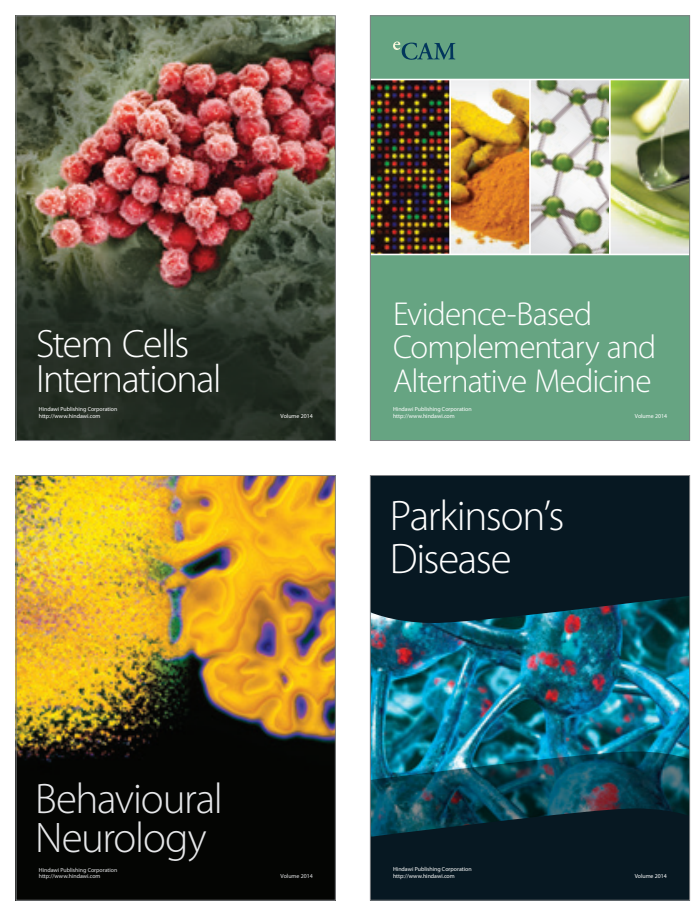

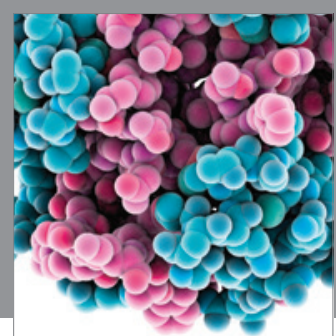

Journal of
Diabetes Research

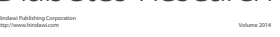

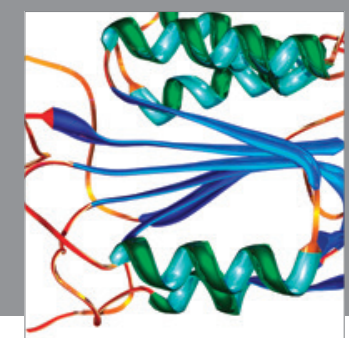

Disease Markers
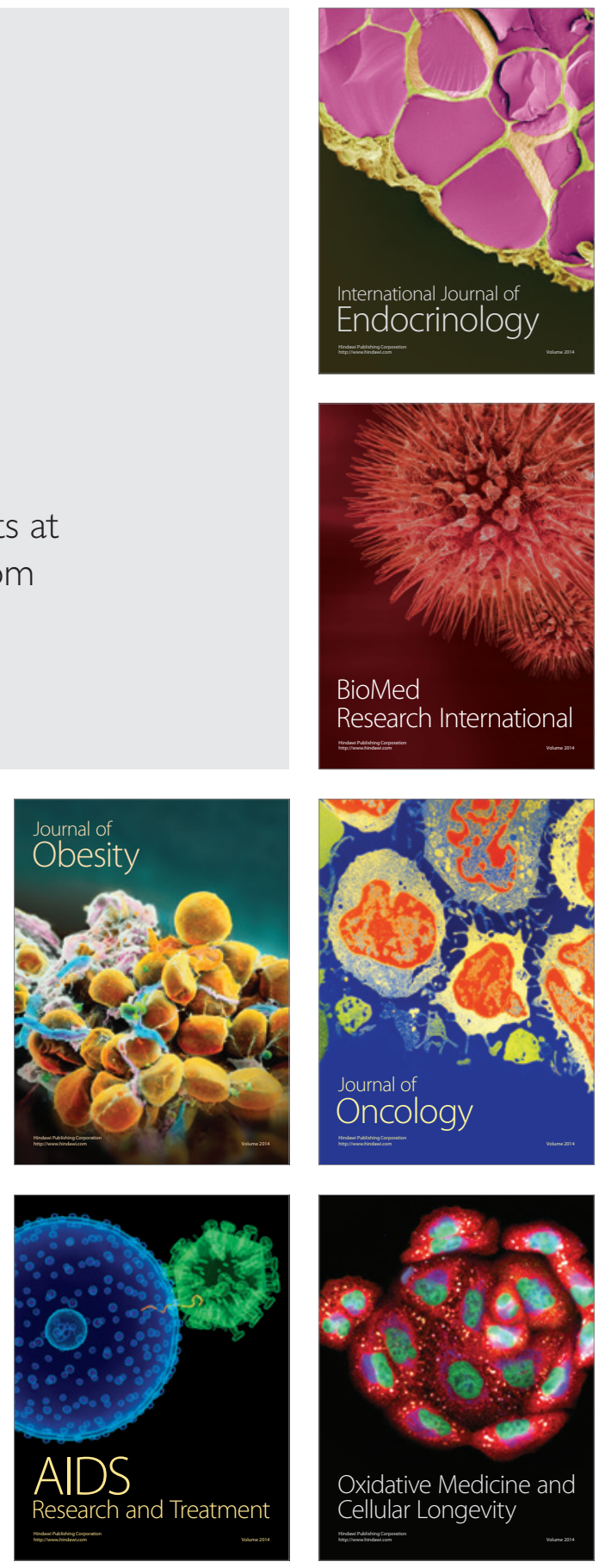